\title{
Anesthetic Management of Patient With Dravet Syndrome: A Case Report
}

\author{
Naohiro Ohshita, DDS, PhD,* Kaname Tsuji, DDS, PhD, $\dagger$ Hiroaki Yoshida, DDS, PhD, $\dagger$ \\ Hiroki Shibata, DDS, PhD, ${ }^{*}$ Yoshiko Matsuda, DDS, PhD, ${ }^{*}$ Yasuo M. Tsutsumi, MD, PhD, $\neq$ \\ and Yoshihiro Momota, DDS, PhD* \\ *Department of Anesthesiology, Osaka Dental University, Osaka, Japan, †First Department of Oral and Maxillofacial Surgery, Osaka Dental \\ University, Osaka, Japan, and $\neq$ Department of Anesthesiology, Tokushima University, Tokushima, Japan
}

Dravet syndrome (DS) is a rare and severe form of epilepsy that begins in infancy. This is particularly burdensome because repeated epileptic seizures lead to cognitive decline. We describe the case of a 12-year-old girl who was diagnosed with DS and was scheduled to have gingival reduction around her mandibular molars. Despite the patient being intellectually disabled, she was able to cooperate somewhat during medical procedures, including intravenous cannulation. Under the assumption that the major problem with anesthesia for DS would be the regulation of body temperature-induced seizures, we used body temperature management equipment to maintain the patient's body temperature during the procedure. We opted for intravenous sedation and administered a total dose of $4.5 \mathrm{mg}$ midazolam throughout the procedure. Anesthesia was completed within 1 hour and 20 minutes without any adverse events. To the best of our knowledge, no previous studies have documented the anesthetic management of DS. In this case, no adverse events occurred perioperatively. However, the patient's temperature rose to that which indicated a slight fever despite the use of a standard cooling technique.

Key Words: Dravet syndrome; Epilepsy; Body temperature; Sedation.

$\mathbf{D}^{\prime}$ ravet syndrome (DS) is a severe myoclonic epilepsy of infancy. ${ }^{1}$ Drug resistance is one of the main features of this syndrome, as seizures are often refractory to drug treatment. ${ }^{1}$ DS is a partial epileptic encephalopathy leading to cognitive decline. ${ }^{2,3} \mathrm{We}$ document a case of anesthetic management via intravenous (IV) sedation of a patient with DS and comorbid intellectual disability.

\section{CASE PRESENTATION}

A 12-year-old girl (height, $139.5 \mathrm{~cm}$; weight, $27.3 \mathrm{~kg}$ ) diagnosed with DS was scheduled for removal of gingival enlargement under IV sedation. At the time of the procedure, the patient's gingival enlargement was causing malocclusion (Figure). Although her regular

Received July 23, 2018; accepted for publication December 11, 2018. Address correspondence to Dr Naohiro Ohshita, Department of Anesthesiology, Osaka Dental University, 1-5-17 Otemae, Chuo-ku, Osaka 540-0008, Japan; oshita@cc.osaka-dent.ac.jp.

Anesth Prog 66:156-158 2019 | DOI 10.2344/anpr-66-02-03

(C) 2019 by the American Dental Society of Anesthesiology dentist had only opted for a follow-up examination, her parents consulted us for a second opinion.

Her past medical history included febrile seizures, focal or myoclonic seizures, cognitive impairment, and personality disorder. She had initially begun experiencing febrile seizures soon after birth, followed by focal or myoclonic seizures. She was diagnosed with DS at the age of 8 months. Her epilepsy was often accompanied by status epilepticus (SE), which persisted until the age of 2 years and 3 months. Currently, her seizures are generalized, lasting 2 minutes, and are sometimes treated with a diazepam suppository as rescue medication. Her seizures were occurring on average 4 times per year, but had occurred 8 times in the last year and, of note, 3 times over the course of a few months prior to surgery. The patient was initially diagnosed with partial seizures, which evolved into her current-day secondary generalized seizures. Her epilepsy is treated with topiramate, potassium bromide, and sodium valproate. The findings on preoperative workup were normal, although the topiramate caused her to develop a sweating disorder that made it difficult for her to control her body temperature. It was also the likely cause of the gingival enlargement. 


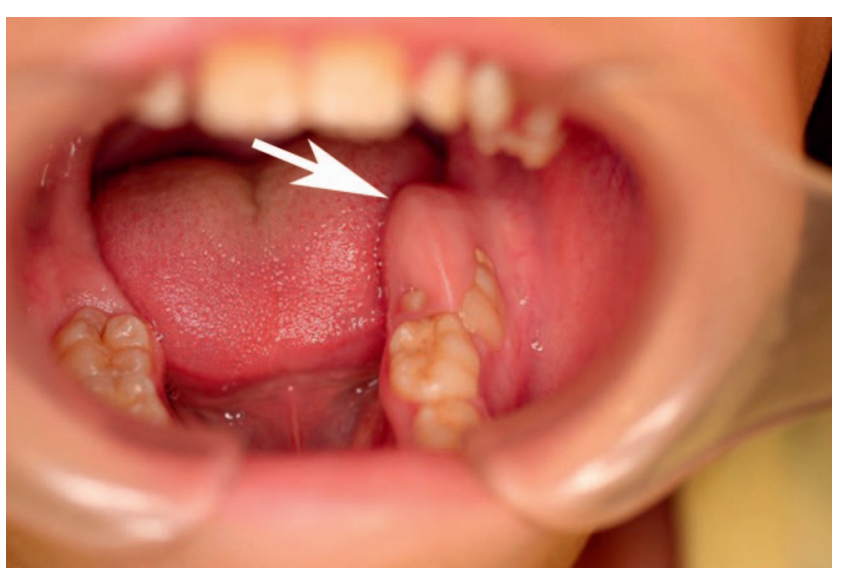

Gingival enlargement in a 12-year-old girl with Dravet syndrome, covering the mandibular molars, inhibiting normal occlusion.

On the day of operation, the patient arrived at our outpatient clinic accompanied by her mother. Her vital signs included a blood pressure of $100 / 65 \mathrm{~mm} \mathrm{Hg}$, heart rate of 75 beats per minute with normal sinus rhythm, oxygen saturation of $99 \%$ in room air, and a body temperature as measured by axillary thermometer of $36.7^{\circ} \mathrm{C}$. Room temperature and humidity were $25.6^{\circ} \mathrm{C}$ and $56 \%$, respectively. In total, $2.5 \mathrm{mg}$ of midazolam was initially administered, and the Ramsay sedation scale yielded a score of 2 (cooperative, oriented, tranquil). Subsequently, midazolam was infused continuously at a rate of $3.0 \mathrm{mg} / \mathrm{h}(\sim 1.8 \mathrm{mcg} / \mathrm{kg} / \mathrm{min})$. During the operation, the Ramsay score was maintained at level 2. Vital signs were mostly stable, with a systolic blood pressure of $100-120 \mathrm{~mm} \mathrm{Hg}$; a heart rate of $80-90$ beats per minute, except after administration of $1.8 \mathrm{~mL}$ of local anesthetic ( $2 \%$ lidocaine including epinephrine 1:80,000); and an oxygen saturation level of $99-100 \%$ during the invasive treatment. We managed to maintain her ear temperature below $37.4^{\circ} \mathrm{C}$ using body temperature management equipment (Warm Touch; Medtronic). Of the 5 temperature settings (ambient, $34^{\circ} \mathrm{C}, 40^{\circ} \mathrm{C}, 45^{\circ} \mathrm{C}$, $47^{\circ} \mathrm{C}$, and boost) that Warm Touch provides, we used the ambient temperature setting. A total dose of $4.5 \mathrm{mg}$ of midazolam was administered, and the procedure was completed within 1 hour and 20 minutes, free of any adverse events. After surgery, the patient was hospitalized overnight, during which she was accompanied by her mother as a precaution in case of an emergency.

\section{DISCUSSION}

Severe myoclonic epilepsy of infancy was first described in France in 1978. The increasing number of diagnosed cases resulted in more accurately defined characteristics, and the name of the condition was changed to DS. ${ }^{1}$ DS is associated with mutations in the voltage-gated sodium channel gene, $S C N 1 A$, in $75 \%$ of cases. ${ }^{1,2,4-6}$ In vitro, epilepsy in DS is supposedly caused by increased sodium currents and increased sodium current density, inducing spontaneous bursting and epileptic-like hyperexcitability in DS patient-derived neurons. ${ }^{6}$

In Japan, DS is estimated to affect 3000 individuals, with an incidence rate of 1 in 40,000 in the general population. ${ }^{3,4}$ The mortality rate secondary to SE is high, and unconscious patients are often rushed to the emergency room. ${ }^{1}$ In this case, the patient's mother had saved her from numerous crises with rectal diazepam. A comprehensive literature search revealed that sudden unexpected death in epilepsy in DS is the likeliest cause of nearly half of all mortality cases. ${ }^{7}$ Mean age at death is $8.7 \pm 9.8$ years. $^{7}$ Drug resistance is one of the main features of this syndrome, but for this patient, seizure control was relatively good with daily administration of drugs. However, her seizures had occurred 8 times over the course of the past year. In 2011, stiripentol, a GABA-ergic antiepileptic drug with a specific FDA indication for DS when coadministered with clobazam, and levetiracetam were recommended as new antiepileptic drugs that could be efficacious in shortening convulsive seizures of longer duration and avoiding SE in DS. ${ }^{1}$ A recent article showed that a cytochrome P450 isoform inhibitor was effective as an adjunct to existing antiepileptic drug treatments. ${ }^{8}$

A major problem with anesthesia for patients with DS is the regulation of body temperature-induced seizures, because fever and photosensitivity associated with pattern sensitivity can easily induce seizures in DS patients. ${ }^{1,3,4}$ In this patient, seizures had previously been induced by a fever $\left(>38^{\circ} \mathrm{C}\right)$ as well as by the photic stimulation of a mobile game. In addition, dysregulation of her body temperature was influenced by one of her medications for epilepsy, topiramate, and she was unable to regulate body temperature because of hypohidrosis. We used body temperature management equipment to cool her during the procedure for about an hour, but her temperature still rose to that of a slight fever. It would clearly be more difficult to control the body temperature of a patient with DS during even longer surgeries. It might be more difficult to control under general anesthesia, which suppresses the hypothalamus more than under IV sedation, although temperature is usually decreased without active warming. In our procedure, an unexpected fever arose despite use of a standard forced-air warming machine set to ambient room temperature. With our knowledge regarding preventing heat retention from this case, in the future we would be prepared to use cold IV fluids or ice packs, in addition to standard body temperature 
management equipment, depending on case presentation. Another potential problem during preoperative examination is that of a low platelet count (frequency $38 \%){ }^{4}$ However, in this case, platelets were within the normal limit $(225,000 / \mu \mathrm{L})$.

In theory, dental surgery in disabled patients should be suitable for ambulatory treatment under general anesthesia. However, this form of anesthesia is linked to a number of postoperative complications, including but not limited to nausea and vomiting, fever, malignant hyperthermia, and delayed emergence. ${ }^{9,10}$ It may be preferable to substitute IV sedation for general anesthesia, if possible. Although the outcome under IV sedation is influenced by patient cooperation, our patient was able to understand instructions and remain compliant with her mother's assistance. She thus remained calm and was able to receive treatment without difficulty, making it possible for us to perform this dental procedure under IV sedation. Previous reports have indicated that the use of IV sedation is an appropriate way of managing patients with disabilities, which was successful for this patient. ${ }^{11}$ We chose overnight hospitalization in our dental hospital for additional safety.

Furthermore, we managed her case without using the potentially seizure-inducing nitrous oxide, ${ }^{12}$ choosing to use midazolam instead. Benzodiazepines are widely used to terminate episodes of SE, and their administration is recommended for the management of early stages of SE. ${ }^{13}$ Antiepileptic drugs that are useful for epilepsy in DS include benzodiazepines, valproate, ethosuximide, and bromides. ${ }^{1}$ In this case, midazolam was also effective in preventing tongue dyskinesia.

There are other considerations to note. First, no previous studies have documented the anesthetic management of DS. Although regulation of body temperature is important, as discussed here, other elements may also be important. There may be an optimal season for surgery that varies across patients based on seizure history. For example, following our patient's frequent seizure occurrences, performing surgery in the hot month of August was not ideal. Finally, it is known that some anesthetic drugs are potentially seizure inducing, and consideration should be given to limiting use of these drugs when possible.

\section{CONCLUSION}

We report a case of IV moderate sedation for oral surgery that was successful for a patient with DS presenting with intellectual disability. We note that body temperature may be resistant to cooling during surgery and requires careful monitoring.

\section{ACKNOWLEDGMENT}

The authors thank Dr Shoko Gamoh (Department of Oral Radiology, Osaka Dental University, Osaka, Japan) for help in establishing the appropriate diagnosis.

\section{REFERENCES}

1. Dravet C. Dravet syndrome history. Dev Med Child Neurol. 2011;53(suppl 2):1-6.

2. Dravet C. Terminology and prognosis of Dravet syndrome. Epilepsia. 2014;55:942-943.

3. Dravet C. Addressing the needs of patients and their family: conclusion. Eur J Paediatr Neurol. 2012;16(suppl 1): S18-S20.

4. Ishii A, Watkins JC, Chen D, Hirose S, Hammer MF. Clinical implications of SCN1A missense and truncation variants in a large Japanese cohort with Dravet syndrome. Epilepsia. 2017;58:282-290.

5. Villas N, Meskis MA, Goodliffe S. Dravet syndrome: characteristics, comorbidities, and caregiver concerns. Epilepsy Behav. 2017;74:81-86.

6. Liu Y, Lopez-Santiago LF, Yuan Y, et al. Dravet syndrome patient-derived neurons suggest a novel epilepsy mechanism. Ann Neurol. 2013;74:128-139.

7. Shmuely S, Sisodiya SM, Gunning WB, Sander JW, Thijs RD. Mortality in Dravet syndrome: a review. Epilepsy Behav. 2016;64:69-74.

8. Devinsky O, Cross JH, Laux L, et al; Cannabidiol in Dravet Syndrome Study Group. Trial of cannabidiol for drugresistant seizures in the Dravet syndrome. $N$ Engl J Med. 2017; 376:2011-2020.

9. Marshall SI, Chung F. Discharge criteria and complications after ambulatory surgery. Anesth Analg. 1999;88:508517.

10. Narayan M, Medinilla SP. Fever in the postoperative patient. Emerg Med Clin North Am. 2013;31:1045-1058.

11. Chaushu S, Gozal D, Becker A. Intravenous sedation: an adjunct to enable orthodontic treatment for children with disabilities. Eur J Orthod. 2002;24:81-89.

12. Zier JL, Doescher JS. Seizures temporally associated with nitrous oxide administration for pediatric procedural sedation. J Child Neurol. 2010;25:1517-1520.

13. Perks A, Cheema S, Mohanraj R. Anaesthesia and epilepsy. Br J Anaesth. 2012;108:562-571. 